\title{
Jugando con Dragones: Una experiencia lúdica como introducción a los conceptos filogenéticos en la enseñanza de la biodiversidad
}

\author{
Pablo A. Dinghi \\ Departamento de Ecologia, Genética y Evolución, Facultad de Ciencias Exactas y Naturales, Universidad de \\ Buenos_Aires, Argentina.pablodingbi@gmail.com; pablodinghi@ege.fcen.uba.ar \\ ORCID: https:/ / orcid.org/0000-0002-2895-0863
}

Noelia V. Guzmán

Departamento de Ecología, Genética y Evolución, Facultad de Ciencias Exactas y Naturales, Universidad de Buenos Aires y Consejo Nacional de Investigaciones Cientificas y Tecnológicas, Instituto de Ecología, Genética y Evolución (IEGEBA),Buenos Aires, Argentina.nguzman@ege.fcen.uba.ar

ORCID: bttps:/ / orcid.org/0000-0002-8268-9125

\section{Daniela S. Monti*}

Departamento de Ecología, Genética y Evolución, Facultad de Ciencias Exactas y Naturales, Universidad de Buenos Aires y Consejo Nacional de Investigaciones Cientificas y Tecnológicas, Instituto de Ecología, Genética y Evolución (IEGEBA),Buenos Aires,Argentina.danielamonti@ege.fcen.uba.ar ORCID: https:/ / orcid.org/0000-0003-2579-3767

\section{* Todos los autores aportaron de igual manera al presente trabajo}

[Recibido: 26 Marzo 2019. Revisado: 5 Junio 2019. Aceptado: 19 Agosto 2019]

Resumen: Se propone una actividad lúdica que tiene como fin introducir a los alumnos en el pensamiento de árbol. Los árboles filogenéticos son la forma adecuada de expresar la biodiversidad en el marco teórico de las actuales hipótesis evolutivas, por lo que deberían tomar un lugar central en el entendimiento público de la evolución. Su mala interpretación, sumada a la escasa disponibilidad de material didáctico en idioma español para docentes conduce a la repetición sistemática de errores. De esta manera, los alumnos terminarán por incorporar nociones equivocadas que se verán acrecentadas por los problemas epistemológicos en el aprendizaje de teorías evolutivas. La actividad lúdica como proceso de aprendizaje proporciona herramientas potentes a la hora de adquirir conceptos que requieren abstracción. Los juegos en la enseñanza de ciencias naturales, tanto con enfoques clásicos o más modernos, como el aprendizaje basado en juegos, proporcionan a los jugadores/aprendices una sensación de logro y mejoran la adquisición de conocimientos. En este contexto, la presente propuesta invita a acercarse al trabajocientífico y permite asimilar conceptos abstractos mediante la resolución de acertijos evolutivos. La actividad ofrece los conceptos adecuados para la introducción al estudio de procesos evolutivos y muestra una forma simple de presentarle al alumno la diversidad de la vida en nuestro planeta según los paradigmas actuales dentro del diseño curricular de enseñanza media.

Palabras clave: filogenias; árboles filogenéticos; pensamiento de árbol; biodiversidad; aprendizaje basado en juegos.

Playing with Dragons: A ludic experience as an introduction to phylogenetic concepts in biodiversity teaching

Abstract: The aim of the present paper is to introduce students to tree-thinking through a ludic activity. Phylogenetic trees are the appropriate way to explain biodiversity under the theoretical framework of current evolutionary hypothesis and they should take a central place in public understanding of evolution. Misinterpretation of phylogenies plus the unavailability of didactic material in Spanish for teachers ends up in the reproduction of misunderstandings on the evolutionary theory. The combination of these errors with epistemological problems of evolutionary theories learning generates even more confusion in these subjects. The ludic activity as a learning process provides powerful tools when it comes to acquiring concepts that require abstraction in contents. Educational natural science games, both classical and modern approaches as Game-based 
learning theory, provide a sense of achievement to players/apprentices and they improve knowledge acquisition. In this context, the activity proposed here invites students to think under the scientific method and it allows them to assimilate abstract concepts by the resolution of evolutionary riddles. This activity gives the concepts to introduce scholars to the study of evolutionary processes, and the diversity of life on Earth under the current educational paradigms.

Keywords: phylogenies, phylogentics trees, tree-thinking; biodiversity; game-based learning.

Para citar este artículo: Dinghi P. A., Guzmán N. V., y Monti D. S.(2020) Jugando con Dragones: Una experiencia lúdica como introducción a los conceptos filogenéticos en la enseñanza de la biodiversidad. Revista Eureka sobre Enseñanza y Divulgación de las Ciencias 17(1), $1201 . \quad$ doi: 10.25267/Rev_Eureka_ensen_divulg_cienc.2020.v17.i1.1201

\section{Introducción}

Durante la segunda mitad del siglo XX, el número de estudios que utilizan filogenias para responder preguntas biológicas se ha incrementado hasta constituir, en la actualidad, una prolífera área de estudio (ver citas en Pellens y Grandcolas 2016, tabla 1, p. 4). O’Hara (1997) consideró este desarrollo teórico de la sistemática filogenética como el surgimiento de un nuevo modo de pensamiento al que definió como pensamiento de árbol (definido originalmente en inglés tree-thinking) (O’Hara 1988, Maddison y Maddison 1989, de Queiroz 1992, Doyle y Donoghue 1993, Wake 1994).

Los árboles filogenéticos son la representación más directa del principio de ancestralidad común, núcleo de la teoría evolutiva y por lo tanto, constituyen una herramienta esencial que debería tomar un lugar central en el entendimiento de la evolución (Baum, Smith y Donovan 2005). Según O'Hara (1997), la importancia de aprender a interpretar árboles evolutivos en biología es comparable al aprendizaje de cómo leer mapas en geografía.

En la enseñanza media, si bien se brinda atención a ciertos aspectos macroevolutivos a través de tópicos como el descubrimiento de fósiles, especiación y relación entre especies, estos no se ven acompañados por la comprensión de las filogenias (Meir, Perry, Herron y Kingsolver 2007). A la reciente incorporación de conceptos filogenéticos en los programas de la ciudad de Buenos Aires desde el 2014, se suma la poca oferta de cursos de actualización en el área y la casi inexistencia de material didáctico en idioma español dedicado a los docentes. De este modo, se hace necesario proveerlos de nuevas herramientas que ayuden a introducir a los alumnos en el pensamiento de árbol, evitando la reproducción de errores y malas concepciones que oscurecen el concepto de evolución.

\section{Concepciones erróneas comunes}

Pérez, Gómez Galindo y González Galli (2018) y González Galli, Pérez, Alegre y Escoto (2018) hacen referencia a una serie de dificultades en la enseñanza de la evolución. En particular, Pérez et al. (2018) señalan dos grandes problemas epistemológicos a combatir: el teleológico y el esencialista. En el primero, las nuevas especies adquieren características para contrarrestar algún tipo de desventaja ambiental o simplemente avanzan hacia un diseño de perfección. El segundo implica una visión parcialmente asociada al fijismo, en la cual, si bien se admiten pequeños cambios dentro de las especies, su esencia se considera invariable, conformando compartimentos estancos con límites marcados difíciles de traspasar. Estos problemas están asociados según González Galli y Meinardi (2010) a obstáculos de transversalidad, funcionalidad y conflictividad del conocimiento para la enseñanza de evolución. Los alumnos toman intuitivamente concepciones de dominios diferentes del conocimiento (funcionalidad), las aplican en sus pensamientos evolutivos (transversalidad) y llegan a razonamientos explicativos erróneos de la realidad que los rodea (conflictividad). 
En la actualidad, la degradación de la biodiversidad es un problema mundial, a tal punto que se encuentra en la agenda de las Naciones Unidas como uno de los 17 objetivos de desarrollo sostenible La educación ambiental es un pilar necesario para el desarrollo de actitudes y comportamientos responsables frente a este problema de cara a las próximas generaciones (Ramsey y Hungerford 1989). Para ello es necesario, como primera instancia conocer conceptos básicos de biodiversidad (procesos evolutivos, especiación, taxonomía y filogenias). González García y Salinas Hernández (2004) mostraron el escaso conocimiento por parte de los alumnos sobre biodiversidad y recalcaron la importancia del estudio y conocimiento de las especies. Para obtener un mayor entendimiento del mundo viviente es necesario recurrir a la sistemática biológica como herramienta.

Una vez encarados estos problemas, surge el desafío de enseñar la biodiversidad actual desde una visión evolutiva. Para ello, es necesario recurrir a conceptos filogenéticos que complementen los ya adquiridos sobre evolución de las especies. Gregory (2008) provee una lista detallada de los errores más comunes en la comprensión de árboles evolutivos. A continuación, desarrollaremos algunos de estos:

\section{De simple a complejo - más y menos evolucionado}

Aristóteles en el siglo IV A.C. desarrolló la Scala Naturae (Escala de la Naturaleza), en la cual todas las especies vivas -y entes de origen divino- se encuentran ordenadas de manera lineal, continua y progresiva desde un organismo inferior hacia uno superior o mejorado, pasando por el ser humano hasta llegar a los Dioses del Olimpo en la cima. Estas ideas subsistieron durante siglos debido a la incorporación de los conocimientos aristotélicos por parte de los Escolásticos en la Edad Media.

La idea de continuidad lineal y progresiva entre las especies fue reformulada por Darwin $(1837,1859)$, quien propuso la descendencia común como mecanismo generador de especies y destacó la forma arbórea del proceso evolutivo. Sin embargo, su contribución terminó reemplazando la idea de escala natural por la de escala evolutiva, pues el concepto de evolución siguió siendo entendido como progreso (Ruse 2005). El discurso público y a veces el científico aún hace referencia a especies contemporáneas como más evolucionadas (Fernandez y Sanjosé 2007). Esto no deviene de una mala interpretación de los árboles filogenéticos, sino que constituye un error conceptual muy internalizado, al punto que incluso aquellos que reconocen la naturaleza ramificada del cambio evolutivo pueden interpretarlo como un proceso progresivo y teleológico. En esta narrativa, todas las especies modernas son derivadas o caminos laterales que se abrieron de la línea principal que conduce a los seres humanos.

\section{Similitud vs. relación}

La taxonomía, fundada por Linneo en la primera mitad del siglo XVIII (1758), categorizaba a los organismos sobre la base de la similitud física. A mediados del siglo XX, luego de la teoría sintética de la Selección Natural, no se abandonó este sistema de clasificación. Las primeras metodologías de análisis inferían las relaciones entre especies a partir de sus similitudes morfológicas globales, pues sus autores consideraban que las relaciones ancestro-descendiente no podrían saberse con certeza (Sokal y Sneath 1963). Actualmente, conocemos esta escuela de clasificación como taxonomía numérica o escuela fenética. A partir del desarrollo de la escuela cladística (Hennig 1950), el principal criterio para la clasificación científica es la descendencia común. Aun así, sigue habiendo confusiones asociadas a la semejanza física entre taxa distantes. La similitud morfológica entre especies poco relacionadas puede deberse al fenómeno de convergencia evolutiva. Esto sucede por el modelado morfológico debido a la selección natural causada por presiones ambientales similares en organismos que ocupan 
nichos ecológicos equivalentes (e.g. la forma corporal hidrodinámica de torpedo en distintos grupos de vertebrados acuáticos, como peces, cetáceos y algunos reptiles marinos extintos).

\section{Hermano vs. ancestro}

Un error común consiste en asumir que el ancestro de dos grupos modernos debe ser muy similar o incluso ser uno de ellos. Específicamente, esta mala interpretación se funda en un concepto equivocado del proceso de especiación, pues asume que una especie se transforma gradualmente en otra (anagénesis). Sin embargo, el proceso especiogénico más común es la cladogénesis: la divergencia de dos especies nuevas a partir de una población ancestral. Por ejemplo, la hipótesis que considera a las ballenas y a los hipopótamos como grupos hermanos (e.g. Boisserie, Lihoreau y Brunet 2005) no implica que el ancestro de las ballenas fuera o se pareciera a un hipopótamo o viceversa. De hecho, el registro fósil muestra que las ballenas fósiles no presentan ningún parecido con los hipopótamos modernos (Thewissen y Bajpai 2001, Thewissen y Williams 2002). Cuando se discute la evolución humana, este error se repite. Considerar que el hombre desciende del mono es todavía muy común, tanto en el discurso como en las representaciones gráficas que muestran un cambio progresivo desde el chimpancé hasta el Homo sapiens. Sin embargo, los chimpancés constituyen una rama separada conformada a partir de la cladogénesis de una población ancestral que no era ni humano ni chimpancé.
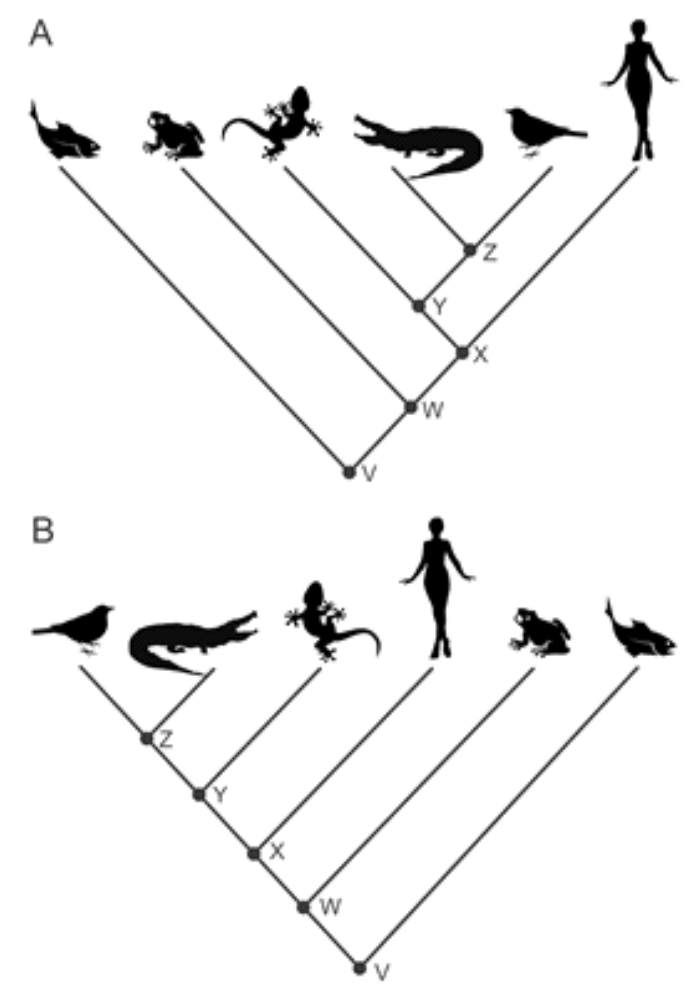

Figura 1. Ejemplos de filogenias que permiten señalar errores comunes en su lectura. A: Cladograma que representa las relaciones entre grandes grupos de vertebrados. B: Mismocladograma con nodos girados. Los ancestros comunes hipotéticos se indican con círculos negros y se nombran con letras (V-Z). Nótese que las relaciones entre taxa están dadas por la cantidad de nodos compartidos y no por la posición de los terminales en el gráfico.

4. Leer dentro del árbol - el problema de los nodos

Si bien los árboles filogenéticos muestran las relaciones entre los taxa (terminales) a partir de los ancestros hipotéticos (nodos), existe una tendencia generalizada a no prestar atención al 
árbol como un todo. Este hecho se ve agravado por la forma de representar los árboles filogenéticos, con los terminales alineados (ya sea de derecha a izquierda o de arriba a abajo) y generalmente, con cierto orden que ubica a un taxón preferido (el considerado por el imaginario popular como el más avanzado) en el extremo derecho o abajo (e.g. humano, Fig. 1A). Algunos errores asociados a este problema son: (1) Considerar más emparentados terminales que están más cerca en el gráfico, en lugar de observar los nodos compartidos por ellos. En la Figura 1A, es erróneo suponer que el gecko está más emparentado con el cocodrilo que con el ave dado que en la ilustración se encuentra al lado del primero y a dos lugares del segundo. La correcta interpretación es que el gecko está igualmente relacionado con ambos dado que comparten un ancestro común (Nodo Y). (2) Considerar que una mayor cantidad de nodos indica menor relación filogenética (Meir et al. 2007). En la Figura 1B, el error consiste en considerar que la rana se halla más relacionada con el pez que con el ave debido a que se encuentra separada de ésta por cuatro nodos internos ( $\mathrm{Z}, \mathrm{Y}, \mathrm{X}, \mathrm{W})$, mientras que del pez está a una distancia de dos nodos (W, V). En realidad, tanto la rana como el ave se encuentran igualmente relacionadas con el pez dado que ambas comparten un ancestro común con el pez (Nodo V). Si bien la actividad propuesta en el presente trabajo no pretende hacer hincapié en estos errores, la misma está diseñada para evitarlos. Por este motivo, se trabajará con una representación filogenética diferente a las que comúnmente se encuentran en las publicaciones científicas o actividades para estudiantes, con los caracteres indicados sobre las ramas y la presencia de ancestros reales, poniendo el foco en todo el árbol y no sólo en los terminales.

\section{Actividad lúdica como proceso de aprendizaje}

Melo Herrera (2014) señala la importancia del juego en la enseñanza de las ciencias naturales como una herramienta esencial en el aprendizaje ya que permite retener representaciones del entorno, aún aquellas introducidas por primera vez ante el individuo (Sutton Smith 1997). Según Melo Herrera (2014, p. 41): "Desde el terreno de la enseñanza y el aprendizaje de las ciencias los resultados de diferentes investigaciones señalan que el juego favorece la creatividad, el espíritu investigativo y despierta la curiosidad por lo desconocido, lo cual es un factor fundamental a la hora de generar preguntas".

Ubicándonos en metodologías innovadoras, el aprendizaje basado en juegos (GBL por sus siglas en inglés, Game-based learning) genera un ámbito en el cual se mejora la adquisición de conocimientos y habilidades y en los casos que éstos involucran la resolución de problemas y desafíos, proporciona a los jugadores/aprendices una sensación de logro y/o reconocimiento (e.g. Qian y Clark 2016, Kirriemuir y McFarlane 2004, McFarlane, Sparrowhawk y Heald 2002, Prensky 2003). Existen numerosos ejemplos que han mostrado cómo el GBL tiende a influir positivamente en las aptitudes y los beneficios cognitivos (Connolly et al. 2012, Dempsey et al. 1994, Hays 2005, Vogel et al. 2006, Wolfe 1997, Young et al. 2012). Un estudio de del Moral, Fernández y Guzmán (2016) mostró que la utilización de videojuegos mejora cualitativamente la adquisición de conocimientos por parte de los alumnos en diferentes áreas, en particular la naturalista dado que los motiva potenciando su aprendizaje, los procesos formativos y la adquisición de saberes. Según Li y Tsai (2013), el GBL en educación científica se apoya en la corriente pedagógica conocida como teoría del conocimiento constructivista. Sus fundamentos teóricos se basan en que la enseñanza y adquisición de saberes se sostienen en una dinámica donde el educando es protagonista e interacciona activamente con el medio. Linares, Gisbert y Garzón (2014)señalan que un enfoque constructivista es un mecanismo en la enseñanza del concepto de evolución biológica es un dispositivo que permite sortear complejidades epistemológicas a la hora de aprender dado que propone actividades para hacer frente a este desafío. El uso de actividades lúdicas en el aula, crea un entorno que estimula a 
los alumnos a construir su propio conocimiento, dejando de lado el aprendizaje memorístico de conceptos. El docente no ocupa un lugar central en la clase y se convierte en un facilitador del proceso de enseñanza - aprendizaje, conduciendo al alumno progresivamente hacia niveles superiores de independencia, autonomía y capacidad para aprender, en un contexto de colaboración que respalda y acentúa los conocimientos adquiridos (Chacón, 2008). La Teoría Sociocultural de Vygotsky (1978) enfatiza la idea de participación proactiva de los niños con el ambiente social que los rodea, agregando que el desarrollo cognoscitivo es fruto del proceso colaborativo entre los actores. Los alumnos desarrollan su aprendizaje mediante la interacción social, realizando tareas en forma compartida que permiten interiorizar las estructuras de comportamiento y pensamiento de la sociedad en las que se desarrollan, seguido de una apropiación de las mismas por parte del individuo. De esta manera, los juegos proporcionan herramientas, un ambiente social adecuado, siendo a la vez un puente entre el conocimiento y el alumno.

A través del juego se invita al alumno a acercarse al trabajo científico, poniendo a prueba -sin explicitar- diferentes hipótesis de relaciones ancestro-descendiente en un contexto más relajado. Así, los alumnos pueden asimilar conceptos teóricos mediante la resolución de un acertijo evolutivo. Cardona (2013) propone renunciar al modelo tradicional de enseñanza de la evolución a través de estrategias innovadoras entre las que se destacan los juegos didácticos. La actividad propuesta usa imágenes, cuya plasticidad interpretativa es más efectiva respecto a la palabra escrita. Gómez Galindo, Gonzalez Galli y Pérez (2017) lo explicitan y proponen actividades usando dibujos en el marco de la modelización de procesos de selección natural.

\section{Objetivo}

La presente experiencia didáctica está diseñada para desarrollarse dentro del aula y está dirigida a alumnos de escuelas medias que hayan explorado -al menos superficialmente- la teoría de selección natural. Su objetivo práctico es usarla como herramienta introductoria para luego extrapolarla a la enseñanza de la biodiversidad, o volver a abordar la misma desde la perspectiva filogenética. El componente lúdico tiene como fin introducir a los alumnos en el pensamiento de árbol, haciendo hincapié en desnaturalizar algunas de las ideas preconcebidas que se traducen en una mala interpretación de las filogenias. Se proporciona un marco teórico para llevar adelante la actividad y una guía para que el docente aborde el tema, así como una serie de preguntas para su discusión. El desarrollo de las ideas propuestas en este trabajo invita al docente a consolidar conceptos curriculares sobre evolución y ofrece herramientas metodológicas modernas aceptadas por la comunidad científica. Es un portal de entrada hacia la enseñanza de la biodiversidad enmarcada en el modelo evolutivo actual, contraponiéndose al modelo enciclopedista clásico de anatomía comparada.

\section{Filogenia de Dragones}

Se buscará que el individuo pueda desarrollar un entendimiento de las relaciones filogenéticas entre taxa y adquiera una interpretación de los agrupamientos válidos bajo el paradigma de la cladística. Los alumnos comprenderán que los grupos deben estar soportados por características derivadas compartidas entre sus miembros (sinapomorfías) e interpretarán por qué éstas son importantes a la hora de entender las relaciones ancestro-descendiente. Siguiendo razonamientos lógicos dentro de la actividad del juego, verán cómo dichas características (en adelante llamadas caracteres) sufren cambios evolutivos desde un estado ancestral o primitivo (en el sentido biológico de primera aparición en el tiempo) a uno derivado. Se estimulará el pasaje intuitivo desde el concepto pre-darwiniano de anagénesis hacia uno clado genético. Esta propuesta puede introducir a la explicación teórica de procesos especio génicos como los de alopatría o vicarianza. 
Se recomienda que sea realizada en forma grupal, fomentando el intercambio de ideas y opiniones entre los educandos. El docente debe procurar que la figura con el árbol filogenético quede a la vista de todos los estudiantes, ubicada de manera central para que tenga un formato de tablero sobre el cual los alumnos puedan participar del juego. Para el desarrollo de la actividad sólo es necesario el concepto de características biológicas heredables según la teoría de selección natural y tener un conocimiento intuitivo de pedigrí o árbol genealógico. Así, a través del pensamiento lógico y de estas herramientas llegarán al análisis de los conceptos filogenéticos anteriormente expuestos.

\section{¿Por qué Dragones?}

\section{1- Universalidad}

Los Dragones como seres mitológicos aparecen en diversas culturas de Oriente y de Occidente. Su universalidad los hace conocidos tanto por la literatura como por filmes, juegos de Rol, de mesa o videogames. Los primeros registros documentados aparecen en las historias épicas clásicas, desde relatos de la Antigua Grecia como la segunda prueba de Hércules matando a la Hidra de Lerna hasta "La leyenda dorada" donde San Jorge vence a caballo a un Dragón [relato de Cocci (1516, en Willis 1993)]. Paralelamente, en el Lejano Oriente aparecen constructos análogos a los dragones indoeuropeos (con origen en China y con cualidades de grandes espíritus o semi-dioses que posteriormente fueron asimilados en Japón y Corea) que hoy en día siguen siendo venerados a través de las tradiciones. En la Edad Media, ambas mitologías tomaron contacto mediante las rutas comerciales entre Oriente y Occidente y posteriormente durante las Cruzadas (Willis 1993). En Mesoamérica existen ejemplos como Quetzalcóatl, "La Serpiente Emplumada", una deidad prehispánica de distintas culturas como la Azteca o Teotihuacana. En los relatos de la actualidad, J.R.R. Tolkien funda su propia mitología con El Hobbit (traducción al español M. Figueroa 1982) y su dragón Smaug. Durante las décadas de los 80 y 90s los dragones constituyen un elemento de gran importancia dentro del universo lúdico gracias al juego de rol Dungeons\& Dragons. También tienen un protagonismo importante en muchas películas, entre ellas Neverending Story (1984, el título en España fue "La historia interminable" y en Hispanoamérica "La historia sin fin"), Dragon heart (1996, en español “Corazón de Dragón”), Harry Potter y el Cáliz de Fuego (2005, basada en el libro homónimo de J. K. Rowling 2000), Eragon (2006) y en 2010 el comienzo de la saga How To Train Your Dragon (en español "Cómo Entrenar a tu Dragón”). A partir del año 2011, con la serie televisiva Juego de Tronos (basada en la saga literaria Canción de Hielo y Fuego de G.R.R. Martin 1996, 1998, 2000, 2005, 2011) alcanzan un público aún más amplio. El conocimiento generalizado de los dragones conlleva una profundidad empírica -consciente o inconscientefuertemente asociada a ciertos sectores de la sociedad, en general jóvenes.

\section{2- Características animales}

Como en otros monstruos mitológicos, las características adjudicadas a los dragones tienenbase en morfologías animales conocidas. En la cultura Occidental, presentan un plan corporal inspirado en los reptiles parecido al de las serpientes, cocodrilos y lagartos con piel escamosa, patas con garras y cola larga. Al igual que en otros seres fantásticos, se suman características pertenecientes a otros grupos de animales como cuernos y alas. En esta actividad su piel cobrará importancia por su origen cromático o mineralógico. Según el tipo de escamas, nos referiremos a dragones blancos, verdes, de oro, cobre, esmeralda o diamante. Por otro lado, los dragones orientales tienen una forma más alargada, serpentiforme y suelen ser no alados. El típico dragón chino está conformado por una quimera de animales: su cabeza porta melena de león y hocico de perro; su cuerpo es de serpiente con garras de águila y puede estar cubierto por pelaje mamiferoide o escamas de pez Koi. Todas estas características permiten navegar la historia evolutiva de los dragones buscando cambios con facilidad. 


\section{3- Seres imaginarios}

La clasificación taxonómica aceptada en la actualidad es generalmente desconocida o malinterpretada por el inexperto. Además, la diversidad animal es compleja para el mapeo de sinapomorfías pues la mayoría de los estudiantes de nivel medio poseen un escaso conocimiento de la morfología y evolución de los distintos grupos. De este modo, el uso de animales mitológicos y universalmente conocidos facilita la comprensión del estudio de características que van apareciendo -o desapareciendo- en la evolución imaginaria de los dragones, sin el sesgo cognitivo que representaría trabajar con grupos de animales reales. En una filogenia los grupos hermanos surgen a partir de un nodo que representa su ancestro común hipotético cuya existencia -o no- es irrelevante para la comprensión de las relaciones de parentesco. Este concepto es una hipótesis ad hoc que sirve para marcar el punto en donde dos grupos divergen. Por ejemplo, en el caso de los mamíferos terios (marsupiales y placentarios) el ancestro común hipotético nos informa que estos derivan de un ancestro terio que poseía las características sinapomórficas comunes a ambos grupos (i.e. el embrión tiene al menos un mínimo período de desarrollo dentro el útero materno). El uso de animales imaginarios permite darle entidad a nodos internos dentro del árbol (ancestros bipotéticos), análogamente a una genealogía, dado que este concepto es más familiar para los alumnos. Sin embargo, este ancestro en las filogenias es un constructo que sirve para entender los procesos especiogénicos por cladogénesis. Luego, cuando se trabaje con taxa reales se podrá revertir esta lógica genealógica hacia la filogenética.

\section{Desarrollo de la actividad}

Se provee de una lámina para imprimir que presenta una historia evolutiva hipotética de los dragones, basada en la separación Oriental-Occidental (Material Suplementario S1). Fueron seleccionadas arbitrariamente distintas características que se explicitan sobre las ramas internas del árbol. En la Figura 2 se muestra esta misma imagen en baja resolución para que el educador pueda seguir los conceptos que se desarrollarán a continuación. También, se dispone de 21 imágenes de dragones para imprimir (Material Suplementario S2, Fig. 3, ilustraciones originales de Melina Lema).

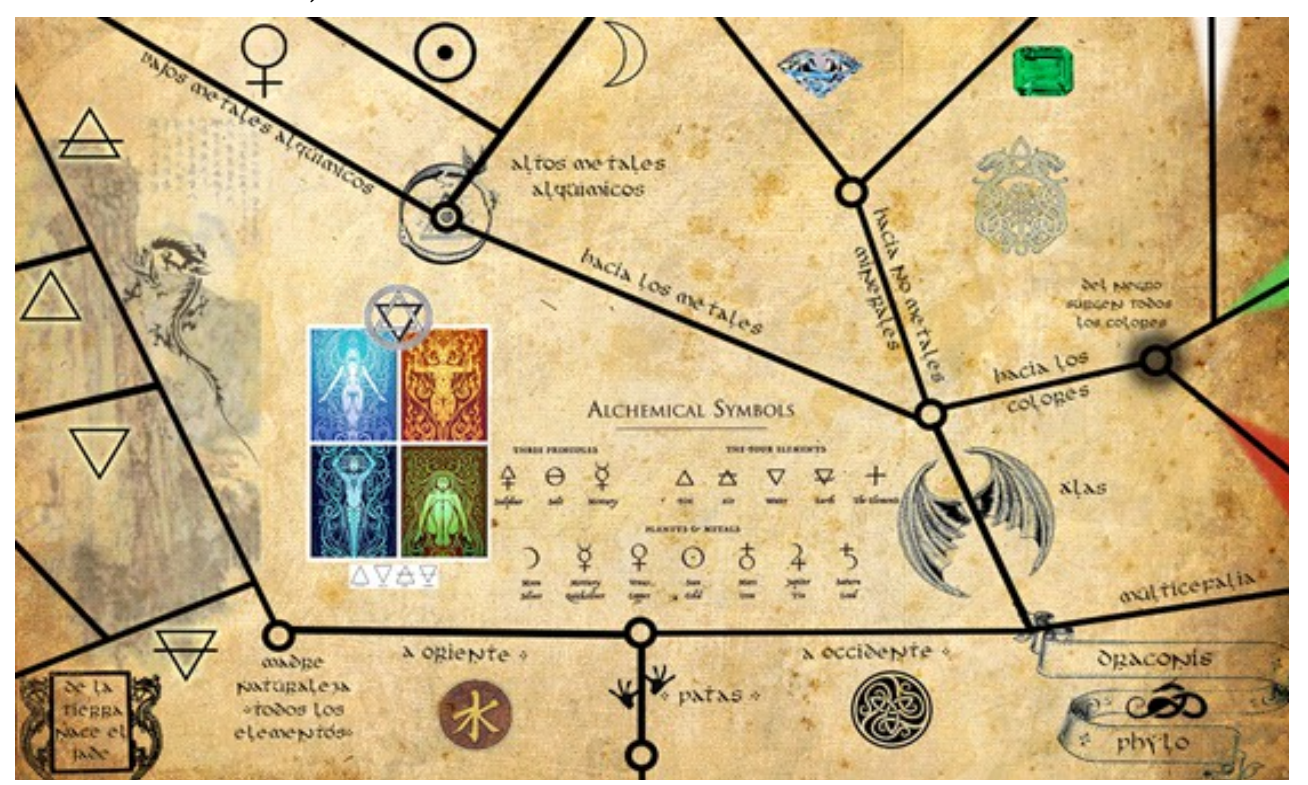

Figura 2. Imagen reducida de la filogenia hipotética de los dragones. Sobre las ramas se indican los cambios en los caracteres durante la evolución de los diferentes grupos. Estos están representados con palabras, símbolos alquímicos o dibujos, referenciados en la misma lámina. Los nodos internos que poseen círculos indican las ubicaciones de los ancestros hipotéticos ilustrados en las cartas. 
El objetivo de la actividad es que los alumnos reconstruyan la historia evolutiva de los dragones. Para ello deberán colocar sobre la lámina (Fig. 2) los 21 dragones en el lugar que corresponda, según las particularidades que observen en las imágenes que se les presentarán y su correlación con las características que van modificándose en la historia evolutiva de éstos. Las ubicaciones posibles se encuentran restringidas al final de cada rama en el margen de la lámina (Fig. 2) y a los círculos internos que representan algunos de los ancestros hipotéticos. Nótese que no todos los nodos internos tienen círculo. El docente deberá hacer hincapié en que cualquier bifurcación denota un ancestro común hipotético, exista o no carta para ubicar en dicho lugar (Fig. 2). El diseño de la lámina prioriza la simbología sobre el lenguaje escrito, dado que fue concebido con el objetivo del aprendizaje a través de alegorías (tratadas como pequeños textos con algo de misterio) y símbolos convencionales de la alquimia. Tanto la plasticidad de la imagen frente a la escritura como lo alegórico permiten al alumno una interpretación más libre y una asociación a lo lúdico (Fig. 2)
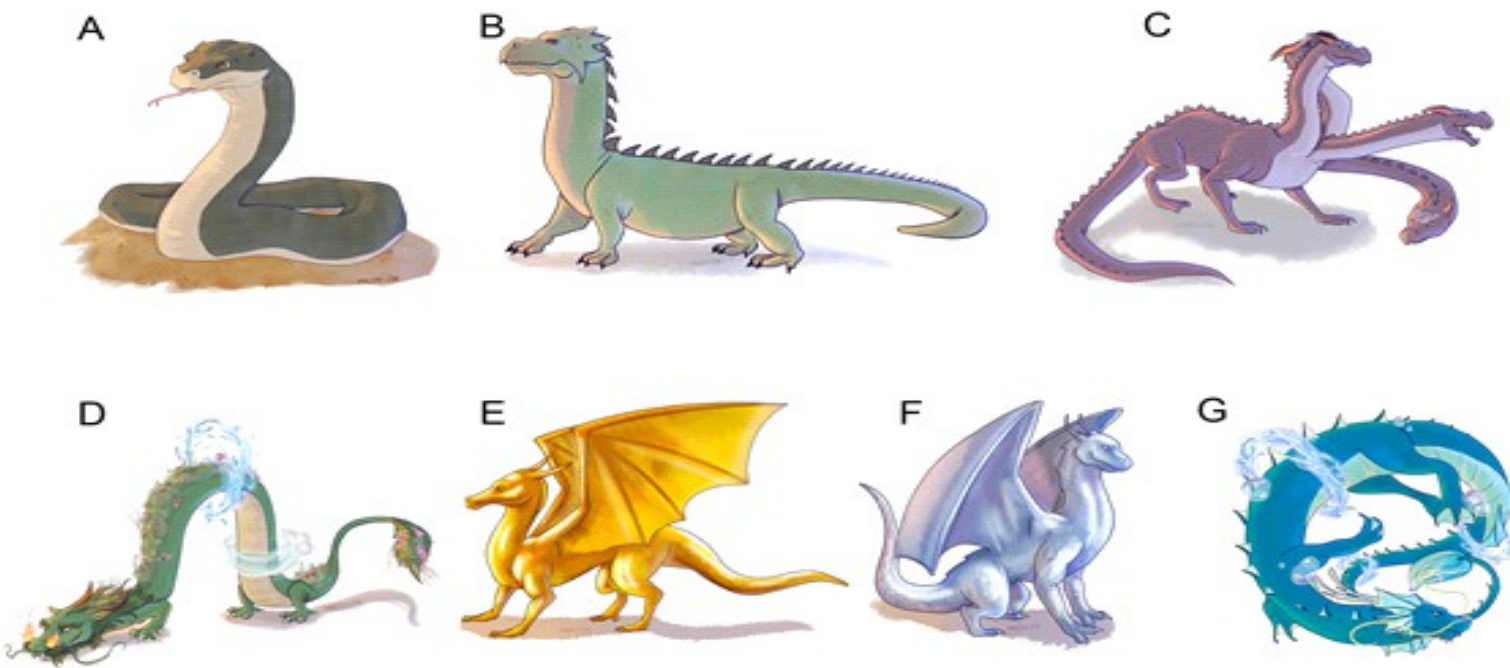

Figura 3. Algunos ejemplos de las cartas a utilizar. A: Ancestro común a todos los dragones. B: Ancestro común a los dragones orientales y occidentales. C: Hidra. D: Dragón oriental que posee todos los elementos, ancestro de todos los terminales orientales. E: Dragón Occidental de Oro. F: Dragón Occidental de Plata. G: Dragón Oriental de Agua. (Ilustraciones originales de Melina Lema, mail: entreplantasypinceles@gmail.com, Instagram: melmellow97).

Guía para el docente: El docente repartirá las 21 figuras de los dragones para que los alumnos observen y discutan posibles agrupamientos sobre la base de sus características morfológicas. Dado que las alas surgen en una rama que incluye a todos los dragones occidentales excepto a la Hidra, se sugiere como primer paso separar los dragones alados (exclusivamente occidentales, e.g. Fig. 3E, F) de los no alados (e.g. Fig. 3A, B, C, D, G). Entre los dragones sin alas, encontramos las típicas formas orientales y algunas que resultarán difíciles de clasificar, entre ellas los ancestros anteriores a la divergencia Oriental-Occidental y la Hidra (Fig. 3 A, B, C).

Los dragones orientales están separados por los elementos: aire, agua, fuego y tierra (este último presenta una divergencia de la que surge el dragón de jade). Su ancestro posee todos los elementos y simboliza a la madre naturaleza a través de las flores que crecen sobre su piel (Fig. 3D). Luego, los diferentes dragones descendientes estarán asociados a un solo elemento cada uno (e.g. Fig. 3G). Nótese que el ancestro posee más características que los descendientes por lo que el docente debe enfatizar que los procesos evolutivos no sólo implican aumento de complejidad en la naturaleza, sino que, en muchos casos, implica simplificación o pérdida de 
estructuras. Algunos ejemplos en la naturaleza son: pérdida de capacidad del vuelo en aves (ñandúes, perdices), pérdida de extremidades en serpientes y atrofia de ojos en animales subterráneos (topos, cecilias).

Entre los dragones occidentales, la primera divergencia se da entre los dragones alados y la ya mencionada Hidra que no posee alas, ésta además presenta una autoapomorfía (i.e. carácter propio y no compartido): la multicefalia. Entre los dragones alados podemos reconocer tres grandes grupos: los cromáticos, los de minerales no metálicos y metálicos. Las figuras permiten separar estos tres grupos por texturas con facilidad. Es importante destacar que en este caso se adquieren características novedosas, al contrario de lo que veíamos en los dragones orientales. Tomando como ejemplo al Dragón Negro (estado ancestral ausencia de color), su descendencia presenta los estados derivados correspondientes a distintos colores (Dragones Rojo, Verde y Blanco). Nuevamente, se sugiere que el docente cite ejemplos de estructuras más o menos complejas que aparecen en la historia evolutiva de animales reales: alas en insectos, garras especializadas en animales cavadores (topos, oso hormiguero, pangolín), lengua muy modificada en camaleones, entre otros. Para los dragones minerales tendremos un ancestro generalizado sin distinción obvia, mientras que sus descendientes tendrán características conspicuas. Dentro de los dragones metálicos nótese que el de oro y el de plata están más relacionados entre sí que con el de cobre, lo que permite profundizar en el concepto de grupos hermanos (i.e. grupos que derivan de un ancestro común inmediato).

Cierre de actividad: Finalizada la actividad se sugiere una discusión abierta sobre las relaciones filogenéticas. Para ello se propone al docente la siguiente guía orientativa:

1- Todos los dragones actuales poseen patas. ¿Eso quiere decir que el ancestro de todos ellos también las tenía? Explica que pudo haber pasado.

2- ¿Qué características crees que puede haber tenido el ancestro común a todos los dragones alados? ¿Cómo te lo imaginas?

3- ¿Los dragones metálicos actuales se encuentran todos igualmente emparentados entre sí? ¿Cómo crees que hubiera sido el ancestro común de los dragones de oro y de plata?

4- ¿Qué relaciones tienen los dragones orientales de agua, fuego y aire? ¿Algunos elementos son más cercanos entre sí que con otros?

5- ¿De qué color eran las escamas del ancestro de todos los dragones cromáticos? Imagina procesos por los cuales pudieran cambiar los colores de las escamas a los dragones cromáticos actuales.

6- Se dice que los dragones metálicos viven en regiones muy tormentosas y con muchos rayos. Propone una utilidad para sus escamas metálicas que los beneficie en estos ambientes.

7- Los dragones de esmeralda y diamante son los que poseen la piel más dura de todos, imagina en qué los podría beneficiar esa característica y en qué los podría perjudicar. Ayuda: Se dice que son muy mansos, no suelen atacar y no son buenos voladores. ¿Conoces animales acorazados en la naturaleza? ¿Presentan comportamientos similares a los mencionados aquí? ¿Cómo crees que la selección natural ha actuado sobre este tipo de animales?

8- Comenta los cambios evolutivos desde el ancestro común a todos los dragones hasta llegar a la Hidra. ¿Cuántas características novedosas aparecieron? ¿Podrías hacer lo mismo contando qué sucedió en la evolución de los dragones hasta llegar al dragón verde?

9- ¿Los dragones actuales son todos igualmente evolucionados?

10- Imagina que tienes una charla con Charles Darwin y le cuentas tu descubrimiento de la filogenia de dragones. Una vez que te escucha con suma atención te pregunta: ¿Por qué crees 
tú que los dragones orientales y occidentales son tan diferentes? ¿Cómo podrías explicárselo? Ayuda: Parece ser que los dragones se separaron a occidente y a oriente hace miles de años. Los de occidente se instalaron en zonas de alta montaña mientras que los de oriente en zonas bajas, más tranquilas.

\section{Evaluación de la propuesta}

Esta experiencia didáctica se puso a prueba en diferentes ediciones (2017 y 2018) de dos eventos (Semana de la Biología y Exactas en la Noche de los Museos) regularmente organizados por la Facultad de Ciencias Exactas y Naturales, Universidad de Buenos Aires. La Semana de la Biología consiste en jornadas abiertas, libres y gratuitas, dirigidas especialmente a escuelas de nivel medio en las que se muestran las experiencias de los investigadores y donde se desarrollan contenidos educativos relacionados con las líneas de investigación de la facultad. Por otro lado, Exactas en la Noche de los Museos es un evento de carácter similar, pero dirigido a un público más diverso.

\section{Metodología}

La experiencia didáctica se llevó a cabo durante tres días dentro del evento denominado Semana de la Biología (ediciones 2017 y 2018), en la estación demostrativa El Árbol de la Vida. En la misma se presenta una serie de actividades que tienen como objetivo explicar, a través de juegos: qué son, cómo se construyen y para qué sirven las filogenias. Si bien el público que participó en la mostración fue diverso, para su evaluación se registraron los resultados de alumnos de cuarto y quinto año de escuelas medias de la Ciudad Autónoma de Buenos Aires, y su equivalente (quinto y sexto año) en Provincia de Buenos Aires (esto incluye un rango etario entre 16 y 18 años considerando una escolaridad sin recusar).

Los alumnos fueron separados en grupos de no más de 15 individuos, acompañados por su docente, para que todos pudieran tener acceso visual a la lámina, manipular el material y participar interactivamente. Para cada grupo, la mostración fue dividida de tres partes: una primera etapa de diagnosis del conocimiento previo, una de desarrollo de la actividad y una final de evaluación de contenidos adquiridos. La duración total de la experiencia fue de entre 20 a 25 minutos.

Diagnosis: al arribar a la estación, los alumnos fueron recibidos por un expositor-divulgador que brindó una charla de 5 minutos cuya finalidad fue introducirlos a la actividad y evaluar su conocimiento previo del tema. Durante esta etapa, un investigador registró la información con la que arribaban los educandos. Se realizaron una serie de preguntas abiertas respecto al tema a tratar basándose en imágenes populares sobre evolución y filogenia (algunas con conceptos erróneos implícitos, ver Fig. 4).

Desarrollo: la filogenia impresa se ubicó en una mesa redonda de manera tal que pudiera ser rodeada por los espectadores desde todos los ángulos y las cartas se ubicaron dispersas sobre la mesa accesibles a todos. La actividad se expuso tal como fue propuesta en la sección Guía para el docente, con una duración de 15 minutos aproximadamente. Mientras los participantes resolvían el acertijo, se explicaron conceptos básicos como estado ancestral y estado derivado de un carácter. Al colocar las figuras en los nodos internos del árbol, se hizo referencia al concepto de ancestralidad. Se destacó la diferencia entre filogenia y genealogía y se explicó el concepto de ancestro común hipotético, asociándolo al proceso de cladogénesis.

Evaluación: finalizada la actividad, el expositor-divulgador realizó un intercambio con el alumnado de 5 a 10 minutos (según el interés de cada grupo). Se realizaron preguntas al azar del cuestionario incluido en este trabajo (exceptuando aquellas de desarrollo) y se abordaron las Concepciones erróneas comunes antes mencionadas. 


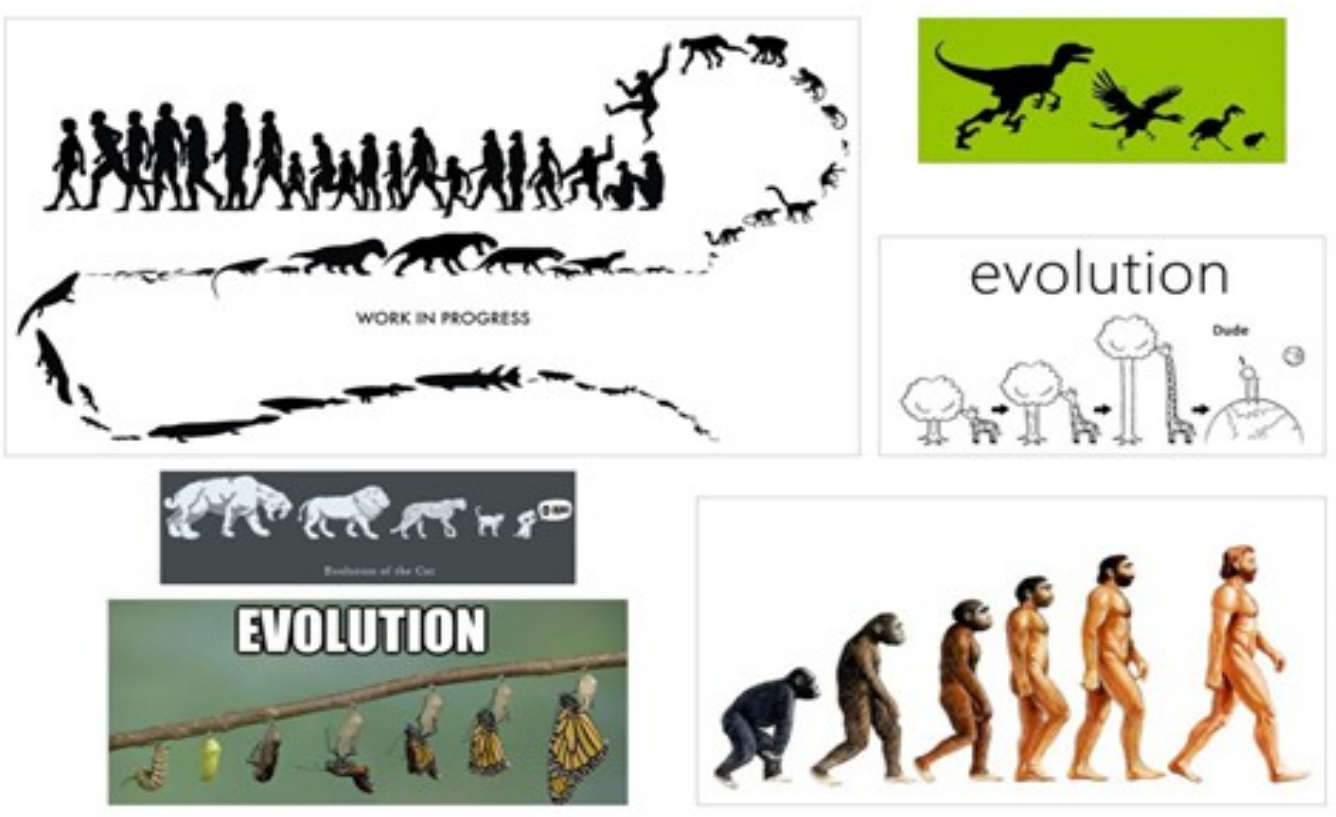

Figura 4. Ejemplos de concepciones erróneas utilizados durante la experiencia obtenidas de internet, utilizando como palabra clave evolución (o evolution).

\section{Resultados}

Diagnosis: durante cada día de las jornadas se recibió en promedio 12 grupos, alcanzando un total de 75 en ambos años. El diagnóstico inicial mostró que la mayoría de los alumnos carecían de conocimientos previos sobre filogenias. Exceptuando grupos puntuales, desconocían dicho término o su representación mental y sólo uno o dos individuos por grupo mostraron nociones aproximadas más relacionadas a las genealogías.

Desarrollo: todos los grupos lograron resolver el juego en el tiempo estipulado. Como primera aproximación desde lo lúdico, esta experiencia resultó ser altamente aceptada y generadora de interrogantes. La temática resultó atrayente por tratarse de animales mitológicos que se encuentran por fuera de los lineamientos típicos en el abordaje de temáticas en ciencias naturales. Los alumnos se mostraron motivados por el desafío de colocar las imágenes sobre la lámina y trabajaron en forma colaborativa para alcanzar el objetivo. Además, con preguntas buscaron extrapolar a la naturaleza los conceptos presentados en el juego.

Evaluación: al finalizar la actividad, el total de los grupos logró diferenciar el concepto de filogenia del de genealogía y comprender el modelo arborescente evolutivo. A través de las imágenes erradas utilizadas en la diagnosis (Fig. 4), se verificó el reemplazo del modelo anagenético de especiación por el cladogenético. Cualitativamente se pudo observar que dentro de los grupos, la mayoría de los alumnos lograron desmitificar el concepto de evolución como progreso y pudieron entender que no necesariamente dos especies similares en su morfología se encuentran más emparentadas entre sí que con una tercera (e.g. Dragón Verde vs. Dragón Esmeralda, Hidra vs. dragones orientales). Los alumnos lograron leer la filogenia correctamente, estableciendo las relaciones evolutivas a partir de las ramificaciones del árbol, donde reconocieron grupos hermanos cada vez más abarcadores.

\section{Conclusiones}

La actividad planteada logró abordar los errores mencionados en la introducción del presente artículo desde un enfoque pedagógico, basado en el pensamiento lúdico y en el trabajo en equipo, lo cual acrecentó notablemente la incorporación de los conceptos a enseñar. De 
manera implícita, esta propuesta resultó efectiva para evaluar las relaciones evolutivas considerando al árbol completo. Se mostró que un árbol filogenético no tiene como fin último encontrar a la especie más evolucionada, reforzando la idea de ancestralidad común, donde todas las especies devienen de una historia compartida.

La aplicación de este tipo de metodologías como introducción al estudio de la biodiversidad propone un marco evolutivo y dinámico en su enseñanza, el cual se despega del aprendizaje enciclopedista basado en anatomía/morfología comparada con el cual suele enfocarse este tema.

Los alumnos deberán poseer un mínimo conocimiento previo, el cual es necesario para entender los procesos especiogénicos involucrados. Más allá de ser una limitante, resulta un incentivo para el docente, promoviendo una reformulación en el dictado de los contenidos curriculares. Desde nuestro punto de vista, este es un aporte a la enseñanza de la biodiversidad, el cual tendría que ser acompañado por otros contenidos que consoliden conceptos y amplíen el conocimiento de la biodiversidad. La misma debe ser entendida integralmente, con una buena base evolutiva y sus desprendimientos epistemológicos en saberes como la genética y el estudio del medio ambiente.

Esta experiencia mostró ser un buen marco de presentación al estudio de problemas referidos a procesos evolutivos. El valor principal que encontramos es que introduce a los alumnos al tratamiento adecuado de la diversidad de la vida en nuestro planeta, de una manera atrayente y divertida, según los paradigmas científicos actuales. Permite un enfoque transversal, uniéndose a otros temas relacionados: la variabilidad y abundancia de especies en nuestro planeta, la importancia de su protección y el rol del ser humano como agente de cambio.

En palabras del biólogo evolutivo Theodosius Dobzhansky: "Nada tiene sentido en biología si no es a la luz de la evolución".

\section{Agradecimientos}

Los autores quieren agradecer especialmente a la ilustradora Melina Lema (entreplantasypinceles@gmail.com, Instagram: melmellow97) por su desinteresada colaboración. Queremos agradecer: a la Facultad de Ciencias Exactas y Naturales, Universidad de Buenos Aires por brindarnos el espacio para llevar a cabo estas actividades y al Instituto de Ecología Genética y Evolución de Buenos Aires (UBA-CONICET) por su apoyo; a los revisores anónimos cuyos aportes enriquecieron significativamente este trabajo y, finalmente, a todos los alumnos y docentes por sus comentarios e inquietudes que incentivaron el desarrollo de este trabajo.

\section{Referencias}

Baum D. A., Smith S. D., Donovan S. S. (2005) The tree-thinking challenge. Science 310 (5750), 979-980.https://doi.org/10.1126/science.1117727

Boisserie J. R., Lihoreau F., Brunet M. (2005) The position of Hippopotamidae within Cetartiodactyla. Proceedings of the National Academy of Sciences 102 (5), 1537 1541.https://doi.org/10.1073/pnas.0409518102

Cardona, 2013. Diseño de una unidad didáctica para la enseñanza de la evolución. Tesis para obtener el grado de Magister en la enseñanza de las ciencias exactas y naturales. Facultad de Ciencias de Medellín, Colombia.

Chacón, P. 2008. El Juego Didáctico como estrategia de enseñanza y aprendizaje ¿Cómo crearlo en el aula? Nueva Aula Abierta 16 (5), 1-8.

Connolly T. M., Boyle E.A., MacArthur E., Hainey T., Boyle J. M. (2012) A systematic literature review of empirical evidence on computer games and serious games. Computers \& Education 59 (2), 661-686.https://doi.org/10.1016/j.compedu.2012.03.004 
deQueiroz K. (1992) Review of Principles of Systematic Zoology, 2 edition, by E. Mayr and P.D. Ashlock. Systematic Biology 41, 264-266.

Darwin C. (1837) Notebook B, 36-74.

Darwin C. (1959) On the origin of species by means of natural selection, or the preservation of favoured races in the struggle for life. London, UK: Murray.

del Moral M.E., Fernández L.C., Guzmán A.P. (2016) Proyecto Game To Learn: Aprendizaje Basado en Juegos para potenciar las inteligencias lógico matemática, naturalista y lingüística en Educación Primaria.Píxel-Bit. Revista de Medios y Educación 49, 177 - 193.

Dempsey J.V., Rasmussen K., Lucassen B. (1994) Instructional gaming: implications for instructional technology. En Paper presented at the Annual meeting of the association for educational communications and technology, Nashville, TN., 16 - 20 de febrero.

Doyle J. A., Donoghue M. J. (1993) Phylogenies and angiosperm diversification. Paleobiology 19 (2), 141-167.https://doi.org/10.1017/S0094837300015840

Fernández J. J., Sanjosé V. (2007) Permanencia de ideas alternativas sobre Evolución de las Especies en la población culta no especializada. Didáctica de las ciencias experimentales y sociales 21, 129-149.

Gómez Galindo A., González Galli L., Pérez G. (2017) ¿Qué aportan los dibujos a la comprensión de los significados de las explicaciones de las explicaciones de los estudiantes en biología evolutiva? Enseñanza de las Ciencias $N^{o}$ extraordinario, 4307-4312.

González Galli L., Meinardi E. (2010) Revisión del concepto de obstáculo a partir de la investigación sobre la enseñanza y el aprendizaje del modelo de evolución por selección natural. IX Jornadas Nacionales y IV Congreso Internacional de Enseñanza de la Biologia, Tucumán, Argentina, 7-10 octubre.

González Galli L. M., Pérez G. M., Alegre C. K., Escoto S. J. (2018) Explicaciones, concepciones y obstáculos sobre el origen de las especies en estudiantes de Escuela Secundaria de Argentina. Ciência $\nLeftarrow E$ Eucação (Bauru) 24 (3), 741-758.

González García F., Salinas Hernández, I.S. (2004) Conocimientos y concepciones sobre biodiversidad en alumnos de educación secundaria. Revista de Educación de la Universidad de Granada 17, 177-188.

Hays R. T. (2005) The effectiveness of instructional games: A literature review and discussion (No. NAWCTSD-TR-2005-004). Naval Air Warfare Center Training Systems Div Orlando Fl.

Hennig W. (1950) Grund zügeeiner Theorie der phylogenetischen Systematik, Berlin: Deutscher Zentralverlag.

Kirriemuir J., McFarlane A. (2004) Literature review in games and learning (Vol 8). Bristol, UK: Futurelab. Li M. C., Tsai C. C. (2013) Game-based learning in science education: A review of relevant research.Journal of Science Education and Technology 22 (6), 877 898.https://doi.org/10.1007/s10956-013-9436-x

Linares M., Gisbert J., Garzón A. (2014). Propuestas didácticas para tratar el origen y evolución de los seres vivos usando recursos TICs y desde una visión constructivista del conocimiento. Congreso Iberoamericano de Ciencia, Tecnología, Innovación y Educación, Buenos Aires: Argentina 12-14 noviembre.

Linneo C. (1758) Systemanatura: per regnatrianaturac, secundumclasses, ordines, genera, species, cum characteribus, differentiis, synonymis, locis.Tomo 1.Editio X Reformata.Holmix (Estocolmo): Impensis Direct LaurentiiSalvii. Biodiversitas Heritage Library. 
Maddison W. P., Maddison D. R. (1989) Interactive analysis of phylogeny and character evolution using the computer program MacClade. Folia primatologica 53 (1-4), 190202.https://doi.org/10.1159/000156416

Martin G.R.R. (1996, 1998, 2000, 2005, 2011) A Song of Ice and Fire (1-5). New York, USA: Bantam Books.

McFarlane A., Sparrowhawk A., Heald Y. (2002) Report on the educational use of games. Cambridge: Teachers evaluating educational multimedia (TEEM).

Meir E., Perry J., Herron J. C., Kingsolver J. (2007) College students' misconceptions about evolutionary trees. The American Biology Teacher 69 (7), 71-76.

Melo Herrera P. (2014) El juego y sus posibilidades en la enseñanza de las ciencias naturales. Innovación Educativa 14 (66), 41-63.

O’Hara R. J. (1988) Homage to Clio, or, Toward an Historical Philosophy for Evolutionary Biology. Systematic Zoology 37 (2), 142-155. https://doi.org/10.2307/2992272

O’Hara R. J. (1997) Population thinking and tree thinking in systematics. Zoologica Scripta 26 (4), 323-329.https://doi.org/10.1111/j.1463-6409.1997.tb00422.x

Pellens R., Grandcolas P. (2016) Biodiversity conservation and phylogenetic systematics: preserving our evolutionary heritage in an extinction crisis (Vol. 14). Springer.https://doi.org/10.1007/978-3319-22461-9

Pérez G., Gómez Galindo A. A., González Galli L. (2018) Enseñanza de la evolución: fundamentos para el diseño de una propuesta didáctica basada en la modelización y la metacognición sobre los obstáculos epistemológicos. Revista Eureka sobre Enseñanza y $\begin{array}{lllll}\text { Divulgación de las } & \text { Ciencias } & .15 & \text { (2), } & 2102\end{array}$ https://doi.org/10.25267/Rev_Eureka_ensen_divulg_cienc.2018.v15.i2.2102

Prensky M. (2003) Digital game-based learning. Computers in Entertainment (CIE)1 (1), 21. https://doi.org/10.1145/950566.950596

Qian M., Clark K. R. (2016) Game-based Learning and 21 century skills: A review of recent research. Computers in Human Behavior 63, 50-58. https://doi.org/10.1016/j.chb.2016.05.023

Ramsey J.M., Hungerford H. (1989) The effects of issue investigation and action training on environmental behavior in seventh grade students. The Journal of Environmental Education 20(4), 29-34. https://doi.org/10.1080/00958964.1989.9943036

Rowling J. K. (2000) Harry Potter and the Globet of fire. Londres: Bloomsbury.

Ruse M. (2005) The Darwinian Revolution, as seen in 1979 and as seen Twenty-Five Years Later in 2004. Journal of the History of Biology 38 (1), 3-17. https://doi.org/10.1007/s10739-004-6506-1

Sokal R. R., Sneath P. H. A. (1963) Principles of Numerical Taxonomy. Freeman, San Francisco.

Sutton Smith B. (1997) The Ambiguity of Play (2nd ed.). Boston, Ma: First Harvard University.

Thewissen J. G. M., Bajpai S. (2001) Whale Origins as a Poster Child for Macroevolution: Fossils collected in the last decade document the ways in which Cetacea (whales, dolphins, and porpoises) became aquatic, a transition that is one of the bestdocumented examples of macroevolution in mammals. American Institute of Biological Sciences Bulletin 51 (12), 1037-1049. https://doi.org/10.1641/00063568(2001)051[1037:WOAAPC]2.0.CO;2 
Thewissen J. G., Williams E. M. (2002) The early radiations of Cetacea (Mammalia): evolutionary pattern and developmental correlations. Annual Review of Ecology and $\begin{array}{llll}\text { Systematics } & 33 & \text { 73-90. }\end{array}$ https://doi.org/10.1146/annurev.ecolsys.33.020602.095426

Tolkien J. R. R. (1982) El hobbit. Capellades: Minotauro. (Traducción Manuel Figueroa).

Vygotsky L. S. (1978) Mind in Society. Cambridge: Harvard University Press.

Vogel J. J., Vogel D. S., Cannon-Bowers J., Bowers C. A., Muse K., Wright M. (2006) Computer gaming and interactive simulations for learning: A meta-analysis. Journal of Educational Computing Research 34 (3), 229-243. https://doi.org/10.2190/FLHV-K4WA-WPVQHOYM

Wake D. B. (1994) Comparative terminology. Science 265 (5169), 268-269. https://doi.org/10.1126/science.265.5169.268

Willis R. (1993) World Mytlhogy, Flos Sanctorum, Duncan Baird Publishers.

Wolfe J. (1997) The effectiveness of business games in strategic management course work. Simulation \& Gaming 28 (4), 360-376. https://doi.org/10.1177/1046878197284003

Young M. F., Slota S., Cutter A. B., Jalette G., Mullin G., Lai B., Simeoni Z., Tran M., Yukhymenko M. (2012) Our princess is in another castle: A review of trends in serious gaming for education. Review of educational research 82 (1), 61-89.

https://doi.org/10.3102/0034654312436980 